\title{
There Are No New Homometric Golomb Ruler Pairs with 12 Marks or Less
}

\author{
Eric Postpischil and Peter Gilbert
}

\section{CONTENTS}

1. Introduction

2. Definitions and Notation

3. Statement of the Result

4. Elementary Properties

5. Statement of the Algorithm

6. Discussion

7. A Geometric Observation

Bibliography
We describe an exhaustive search that proves the nonexistence of homometric Golomb ruler pairs with 7 to 12 marks. We also give a two-dimensional geometric model for the known family of six-mark homometric ruler pairs.

\section{INTRODUCTION}

Familiar rulers are marked in equal increments. The distance across $n$ segments of the ruler is $n$ units. By contrast, a Golomb ruler [Shearer 1990] has segments of unequal length, and each set of adjacent segments measures a distinct distance. For example, a rule having segments of length one, four and two, in that order, can measure distances of one, two, four, five, six, and seven units.

Is the set of distances measured by a Golomb ruler uniquely defined by the lengths and order of the segments? Or is there a nontrivial pair of rulers that measure the same set of distances? (A pair is trivial if the two rulers are identical or reflections of each other.)

In 1939, S. Piccard published a "theorem" saying that no such nontrivial pairs exist. However, G. S. Bloom gave a five-segment counterexample in 1975, later generalized by Bloom and S. W. Golomb to a two-parameter family (see Section 3). Yovanof [1988] describes these examples and illustrates the error in Piccard's proof, which holds for rulers with fewer than five marks.

By exhaustive computer search, we have determined that there are no other nontrivial pairs with fewer than 12 segments. The program treats rulers symbolically, manipulating linear equations, so it 
can conclusively eliminate the possibility of pairs with specific numbers of segments.

\section{DEFINITIONS AND NOTATION}

Dewdney [1985], Yovanof, and other authors represent rulers as a set of numbers that indicate the locations of marks; the difference between two distinct marks is a distance the ruler can measure. For our purposes, it is more convenient to work with the distances between adjacent marks, called segments. Thus, we regard a ruler with $n+1$ marks as an $n$-tuple of positive numbers $\left(a_{0}, a_{1}, \ldots, a_{n-1}\right)$, each of which is called a segment.

A measurement of a ruler is the sum of one or more adjacent segments. Thus an $n$-segment ruler $A$ can measure the distance from the left edge of the $p$-th segment to the right edge of the $q$-th segment, for all $p$ and $q$ with $0 \leq p \leq q \leq n-1$. We call such an ordered pair $(p, q)$ a block, and let

$$
A_{(p, q)}=\sum_{i=p}^{q} a_{i}
$$

be the distance it measures. (We also use the term block for the corresponding union of consecutive segments.) We let $M(n)$ be the set of blocks $(p, q)$; it has $\frac{1}{2} n(n+1)$ elements.

$A$ is a Golomb ruler if all the $A_{(p, q)}$ are distinct. Two Golomb rulers $A$ and $B$ are homometric, denoted $A \sim B$, if there is a permutation $f$ of $M(n)$ such that $A_{(p, q)}=B_{f((p, q))}$ for every $(p, q) \in M(n)$. Note that $f$ must be unique, since no two measurements coincide. The homometric pair is trivial if and only if $f$ is the identity or comes from reflection, that is, $f((p, q))=(n-q-1, n-p-1)$ for all $(p, q) \in M(n)$.

\section{STATEMENT OF THE RESULT}

In Yovanof's formulation, the known six-mark homometric rulers have marks at

$$
\{0, a, 2 a+b, 2 b-a, 2 b+a, 3 b-a\}
$$

and at

$$
\{0, a, b-2 a, 2 b-2 a, 2 b, 3 b-a\},
$$

for values of $a$ and $b$ that result in Golomb rulers. When represented as segments instead of marks, the ruler pairs form two classes, depending on the order in which the marks fall:

$$
(u, v, 3 u+v, 2 u, 2 u+v) \sim(u, 4 u+v, v, 2 u, u+v)
$$

and

$(u, v, 3 u+v, 2 u+2 v, 2 u+v) \sim(u, 4 u+v, v, 2 u, u+v)$,

where $u$ and $v$ are arbitrary positive values except for the restriction that the $A_{(p, q)}$ are all distinct. The shortest pair of equivalent rulers with integer lengths is obtained when $u$ is 1 and $v$ is 3 in the first class, giving a length of 17 .

There are no nontrivial homometric pairs with eleven or fewer segments, apart from the family just given. We describe the algorithm by which this was proved.

\section{ELEMENTARY PROPERTIES}

Let $A$ and $B$ be homometric rulers. The longest measurement of each ruler is its total length, so

$$
A_{(0, n-1)}=B_{(0, n-1)} .
$$

This is a homogeneous linear equation in the $a_{i}$ 's and $b_{i}$ 's, and each of these variables must be positive. Any equation of two measurements is also homogeneous and linear in these variables.

The second-longest measurement of a ruler must be $A_{(0, n-2)}$ or $A_{(1, n-1)}$, since any other block apart from $(0, n-1)$ is contained in one of these two. By flipping the ruler if necessary, we can stipulate that $(1, n-1)$ measures the second-longest distance. Therefore

$$
A_{(1, n-1)}=B_{(1, n-1)},
$$

which together with $A_{(0, n-1)}=B_{(0, n-1)}$ gives $a_{0}=$ $b_{0}$. We also conclude that $a_{0}<a_{n-1}$ and $b_{0}<b_{n-1}$. 
One more linear equation holds for all pairs of equivalent rulers. The sum of all the measurements must coincide for such a pair:

$$
\sum_{0 \leq p \leq q \leq n-1} A_{(p, q)}=\sum_{0 \leq p \leq q \leq n-1} B_{(p, q)} .
$$

This can also be written

$$
\sum_{i=0}^{n-1}(i+1)(n-i) a_{i}=\sum_{i=0}^{n-1}(i+1)(n-i) b_{i},
$$

since segment $i$ appears in every block $(p, q)$ with $p \leq i$ (giving $i+1$ choices) and $q \geq i$ (giving $n-i$ choices).

\section{STATEMENT OF THE ALGORITHM}

For a fixed permutation $f$ of $M(n)$, the condition that $A_{P}=B_{f(P)}$ for every $P \in M(n)$ gives a set of linear equations in the $a_{i}$ 's and $b_{i}$ 's, which must have positive values. Our strategy is to try to build up a permutation $f$ using a depth-first search, using the corresponding partial system of equations to eliminate possibilities.

\subsection{Search Procedure}

From Section 4, we know that $f$ takes each of the blocks $(0,0),(0, n-1)$ and $(1, n-1)$ to itself. We view $f$ as a subset of the Cartesian product $M(n) \times M(n)$, so that $((0,0),(0,0)) \in f$ and so on. The question is to find what other pairs, besides these three, are in $f$.

For this we keep a context consisting of the following information:

(a) All ordered pairs of blocks known to be in $f$.

(b) All blocks in the domain of $f$ that are not yet assigned, and all blocks in the range of $f$ that are not yet assigned.

(c) A set of linear equations that must be true given the pairs in $f$.

(d) A list of assignments that should not be tried again in collateral branches of the search (see below). (e) A flag indicating whether $f$ is diagonal so farthat is, whether all pairs in (a) are of the form $(P, P)$.

The algorithm consists essentially of a routine that takes the current context, considers one at a time all possible ordered pairs that can be added to $f$ (see Section 5.3), and calls itself recursively for each augmented $f$. This goes on until all elements of the domain and range have been assigned, leading to a homometric pair, or all possibilities are exhausted. Note that if symmetry still holds when all elements have been assigned, the permutation is the identity. This trivial solution is discarded.

The initial context includes the three pairs of blocks mentioned above, their associated equations, and (4.1).

When the routine considers a candidate $(P, Q)$ to be added to $f$, it changes the context as follows:

(a) $(P, Q)$ is added to the set of elements known to be in $f$.

(b) $P$ and $Q$ are removed from the sets of unassigned elements of the domain and range, respectively.

(c) The equation $A_{P}=B_{Q}$ is asserted, and the augmented system is analyzed (Section 5.2). If a forbidden situation is found, the routine fails and the search stops along this branch. If forced equalities are found, the corresponding pairs are added to the set of elements known to be in $f$.

(d) $(P, Q)$ is added to the list of assignments not to be tried again.

(e) If $P \neq Q$ and $f$ is diagonal, $f$ is marked as no longer diagonal and $(Q, P)$ is added to the list of assignments not to be tried again.

The idea behind (d) is this: Suppose the search routine has determined $(P, Q)$ and $\left(P^{\prime}, Q^{\prime}\right)$ as possible next assignments. After $(P, Q)$ is attempted and discarded, $\left(P^{\prime}, Q^{\prime}\right)$ is tried. At this point, deeper calls to the search routine might want to $\operatorname{try}(P, Q)$ again, which is wasteful. Thus each pair that is tested at a certain search depth should be forbidden until we return from that depth. (Once 
we return one level higher, some assignment made prior to the testing of the pair in question is undone, and it makes sense to test the pair again in the new context.)

Step (e) represents an optimization that allows us to ignore the inverse of a permutation that we have already investigated. We can do this because, if a valid permutation maps ruler $A$ to ruler $B$, its inverse maps $B$ to $A$, and we don't care about the order of the two rulers.

\subsection{Analysis of the Equations}

We now spell out item (c) above. The first step is to reduce the system of linear equations to rowechelon form. Independent variables are chosen, equal in number to the rank of the system; the remaining variables are considered dependent.

Next we examine the coefficients of the reduced system. If, in any single equation, all the nonzero coefficients (assumed to be all on the same side) have the same sign, a solution with all variables positive is not possible, and we return with failure.

Next we investigate what relations among measurements are forced by the context. We reduce each measurement (which is a sum of certain variables) to an expression in independent variables only. We compare these reduced expressions with one another, looking for the following cases:

1. Two different blocks in the domain, or two different blocks in the range, have measurements whose reduced expressions coincide.

2. Two different blocks in the domain or range have measurements whose reduced expressions add to zero.

3. An unassigned block in the domain and one in the range have measurements whose reduced expressions coincide.

We return with failure in the first two cases, since all measurements for a ruler must be distinct and positive. In the third case we add the ordered pair of blocks whose measurements coincide to the set of pairs known to be in $f$, and keep going.
The actual comparison is done by successively inserting reduced expressions and their negatives into a sorted tree, so equalities can be detected quickly.

\subsection{Adding New Pairs}

Given the current context, we must figure out a list of possible next assignments $(P, Q) \in M(n)$ that will cover all possibilities for constructing $f$. There are different ways to do this.

One way is to take a fixed $P$ in the set of unassigned domain blocks, and pair it with every $Q$ in the set of unassigned range blocks.

A second way is to think in terms of measurements. In $f$, the unassigned domain block with greatest length will turn out to be paired with the unassigned range block with greatest length. We don't know which unassigned blocks in the domain and range have the greatest length, but we can eliminate some candidates: any block contained in another unassigned block cannot be longest. We can therefore make our list by pairing all maximal unassigned blocks in the domain with all maximal unassigned blocks in the range.

A third possibility is similar to the second, but we use minimal, rather than maximal, unassigned blocks.

Any of these three lists will work. Our program chooses the shortest.

\section{DISCUSSION}

The algorithm we have described was implemented in $\mathrm{C}$ (see Electronic Availability below) and run under the Ultrix operating system on several DEC workstations. It finds the expected solutions in the five-segment case but finds no other solutions for eleven or fewer segments. The eleven-segment case required $2.8 \cdot 10^{7}$ seconds, or 320 days, of CPU time, distributed among nine Mips R2000 processors, and amounting to some $2.5 \cdot 10^{14}$ RISC instructions. The ten-segment case required ten CPUdays, and the nine-segment case under two CPUhours. 
Further refinement of the algorithm, and specifically of the analysis in Section 5.2, may speed up the search and settle the case of rulers with more segments. (For instance, our program does not take advantage of the inequality $a_{0}<a_{n-1}$ proved in Section 4.) However, a different approach must be found in order to obtain a proof that will work with an arbitrary number of marks. The authors are divided as to whether any additional nontrivial pairs of homometric rulers are to be found.

\section{A GEOMETRIC OBSERVATION}

Yovanof described several other models for homometric rulers, such as numbered graphs and polynomials. We introduce another model. Take an equilateral triangle with, for example, vertices at $( \pm 2,0)$ and $(0,2 \sqrt{3})$. Counterclockwise from each vertex, extend a line segment half the length of and parallel to the opposite side. In our example, this creates the points $(-2,2 \sqrt{3}),(3, \sqrt{3})$ and $(-1,-\sqrt{3})$. This set of six points has no reflectional symmetry, but the set of 30 vectors joining pairs of points is equal to the corresponding set for the mirror image obtained by reflection in a vertical line (Figure 1). We call a set with this property autometric.
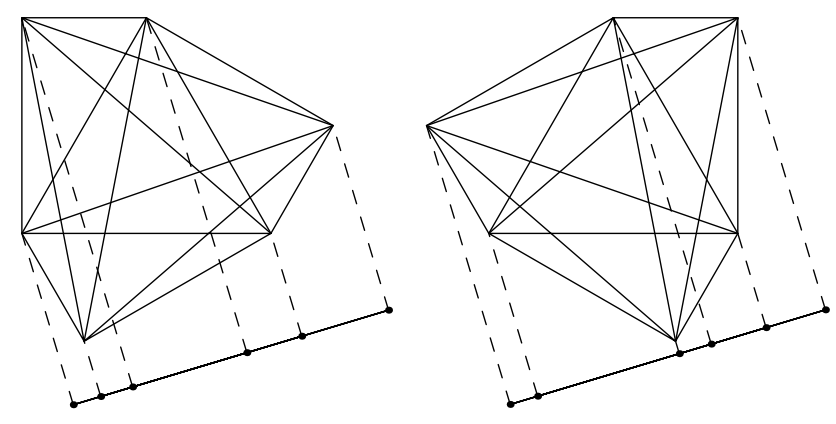

FIGURE 1. An autometric set of points and a corresponding pair of homometric Golomb rulers (see Section 7).
Projecting the six points orthogonally onto any line yields a six-mark Golomb ruler, provided no equal measurements are coincidentally formed by the projection. Moreover, projecting the mirror image onto the same line yields a generally different Golomb ruler. Because the sets of vectors are equal, the projections of the vectors, which are the measurements of the ruler, are equal, yielding homometric Golomb rulers. The reader can check that the six-point autometric set of Figure 1 yields the family of homometric pairs of Section 3 by the following procedure: multiply the points by $-\frac{1}{3} \sqrt{3\left(a^{2}+b^{2}-a b\right)}$, and project onto the line

$$
y=\frac{\sqrt{3}(2 a-b)}{3 b} x
$$

so that a point $(x, y)$ on the plane is taken to the point on the line at a signed distance

$$
-\frac{1}{2} b x-\frac{1}{3} a y \sqrt{3}+\frac{1}{6} b y \sqrt{3}
$$

from the origin.

This leads to the question: Is every pair of homometric Golomb rulers formed by projecting some autometric set?

\section{ELECTRONIC AVAILABILITY}

Upon request, the first author will provide the program by electronic mail or on $3.5^{\prime \prime}$ disks (Unix or DOS format). The program has been tested only under Ultrix.

\section{REFERENCES}

[Dewdney 1985] A. K. Dewdney, "Computer recreations: the search for an invisible ruler that will help radio astronomers to measure the Earth," Scientific American 253(6) (December 1985), 16-26.

[Shearer 1990] J. B. Shearer, "Some new optimum Golomb rulers", IEEE Trans. Information Theory 36 (1990), 183-184.

[Yovanof 1988] G. S. Yovanof, "Homometric structures", Ph.D. Dissertation, University of Southern California, 1988. 
152 Experimental Mathematics, Vol. 3 (1994), No. 2

Eric Postpischil, Digital Equipment Corporation, 6 Hamlett Drive, Nashua, NH 03062 (edp@mv.com)

Peter Gilbert, Digital Equipment Corporation, 291 Merriam Avenue, Leominster, MA 01453

Received June 10, 1993; accepted in revised form September 15, 1994 